\title{
Periventricular Nodular Heterotopias as an Atypical Manifestation of Joubert Syndrome
}

\author{
Himanshu Mishra $M B B S^{1}$, Amit Kumar $M D^{2}$ \\ ${ }^{1,2}$ Department of Radio-diagnosis, IGIMS, Patna, India.
}

\begin{abstract}
Joubert syndrome (JS) is characterized by varying degrees of mid and hindbrain malformations. A thickened superior cerebellar peduncle ("molar tooth sign"), varying degree of cerebellar vermian clefting, and an oddly shaped ("bat-wing") fourth ventricle are essential diagnostic cues on imaging. When JS is associated with renal, ocular, hepatobiliary, or oro-facial abnormalities, the term Joubert syndrome and related disorders (JSRD) is used. We report a classic case of this rare disease in a 5 month old male child who presented to our department for assessment of developmental delay. MRI revealed molar-tooth appearance of midbrain, an abnormally shaped fourth ventricle, and vermian aplasia. Additional findings present in our case were corpus callosum dysgenesis, colpocephaly, generalized cortical atrophy, and periventricular nodular heterotopia. Subsequently, an ultrasound of the abdomen was performed to look for any associated anomalies. It revealed diffuse bilateral echogenic kidneys with attenuated corticomedullary differentiation (likely due to micro cysts in medulla) and few thin-walled peripheral renal cortical cysts.

Callosal dysgenesis, colpocephaly, cortical atrophy and grey matter heterotopias are less common manifestations of JS/JSRD and periventricular nodular heterotopia has been infrequently reported in cases of Joubert syndrome.
\end{abstract}

Key words: Bilateral Echogenic Kidneys, Joubert Syndrome, MRI, Molar Tooth Sign, Periventricular Nodular Heterotopia

Access this article online
Website: https://www.nepjol.info/index.php/NJN
DOI: https://doi.org/10.3126/njn.v18i1.33295
HOW TO CITE
Mishra H, Kumar A. Periventricular Nodular Heterotopias as an
atypical manifestation of Joubert Syndrome. NJNS. 2021;18(1):73-
76.

'ORCID id: 0000-0002-9796-6068

${ }^{2}$ ORCID id: 0000-0003-1231-6179

\author{
Address for correspondence: \\ Dr. Amit Kumar \\ Associate Professor \\ Room No.249, \\ Department of Radio-diagnosis \\ IGIMS, \\ Patna-14, Bihar \\ E-mail: amitmd2008@gmail.com. \\ Phone: +91-8210527229
}

Copyright (C) 2021 Nepalese Society of Neurosurgeons (NESON)

ISSN: 1813-1948 (Print), 1813-1956 (Online)

This work is licensed under a Creative Commons Attribution-Non Commercial 4.0 International License.

\section{Introduction}

Toubert syndrome (JS) is a disease complex which results due to aplasia or hypoplasia of the cerebellar vermis, failure of formation of decussating fibres of superior cerebellar peduncles, and a lack of pyramidal decussations. Marie Joubert and her co-workers were first who described this in 1969. The term Joubert syndrome and related disorders (JSRD) is used when ocular, renal, hepatobiliary, and oro-facial defects are present in addition to the classic features of Joubert syndrome. JS and JSRD are relatively rare diseases, with an estimated incidence of $1: 80,000$ to $1: 100,000$ live births. ${ }^{1}$

Most cases of JS and JSRD follow autosomal recessive inheritance patterns, although rare $\mathrm{X}$-linked $\mathrm{AD}$ gene has also been described cases have been described in literature. ${ }^{2,3}$

Clinical features are variable. Most cases present in infancy and childhood with intellectual/developmental delay, ataxia, hypotonia, irregular breathing pattern, or abnormal eye movements. The affected children may exhibit polydactyly and dysmorphic facial features (such as a broad forehead, arched eyebrows, ptosis, hypertelorism, etc.). ${ }^{1,2,4,5}$

Classic imaging findings, best demonstrated on 


\section{Mishra et al}

magnetic resonance imaging (MRI), are vermian aplasia or hypoplasia, a foreshortened midbrain with thickened superior cerebellar peduncles ("molar tooth sign"), and an oblong or diamond shaped ("bat-wing") fourth ventricle. Additionally, corpus callosum agenesis or dysgenesis and colpocephaly are associated with JS., ${ }^{1,6,7}$ Cortical heterotopias have been infrequently reported in literature, which we describe in our case report. ${ }^{8}$

Renal involvement is noted in about $30 \%$ of cases of Joubert syndrome. It is either Cystic dysplasia with fetal lobulation (Dekaban-Arima syndrome) or Juvenile nephronophthisis type which is seen in our case as diffusely echogenic cortex and medulla due to microcysts in medulla with few peripheral defined cortical cysts as well.

\section{Case Report}

A 5 month old male child presented to the out-patient department with complaints of inability to hold the head at all and two episodes of seizures. He was feeding normally. Birth history revealed a normal vaginal hospital delivery.
He cried immediately after birth. No evidence of perinatal asphyxia was present. Family history was unremarkable.

On examination, the child could not lift the head. Head circumference was within normal limits. Body and limb movements appeared normal. There was no facial asymmetry. Ocular examination was unremarkable. MRI revealed aplastic cerebellar vermis, thickened and elongated superior cerebellar peduncles, and an abnormally shaped fourth ventricle (Fig.1,2).

Corpus callosum was dysgenetic and thinned, with non-visualized splenium. Both the lateral ventricles were dilated, with more prominent posterior horns (colpocephaly) (Fig. 2).

Bilateral periventricular nodular heterotopia (PVNH) was also noted (Fig:3). Generalized cortical atrophy with bilaterally symmetrical widening of subarachnoid spaces was also seen (Fig. 3).

A screening ultrasound scan of the abdomen was performed which revealed thin-walled few peripheral small cortical cysts in both kidneys with homogeneously echogenic renal cortex and medulla due to microcysts in medulla region. Other organs appeared normal.

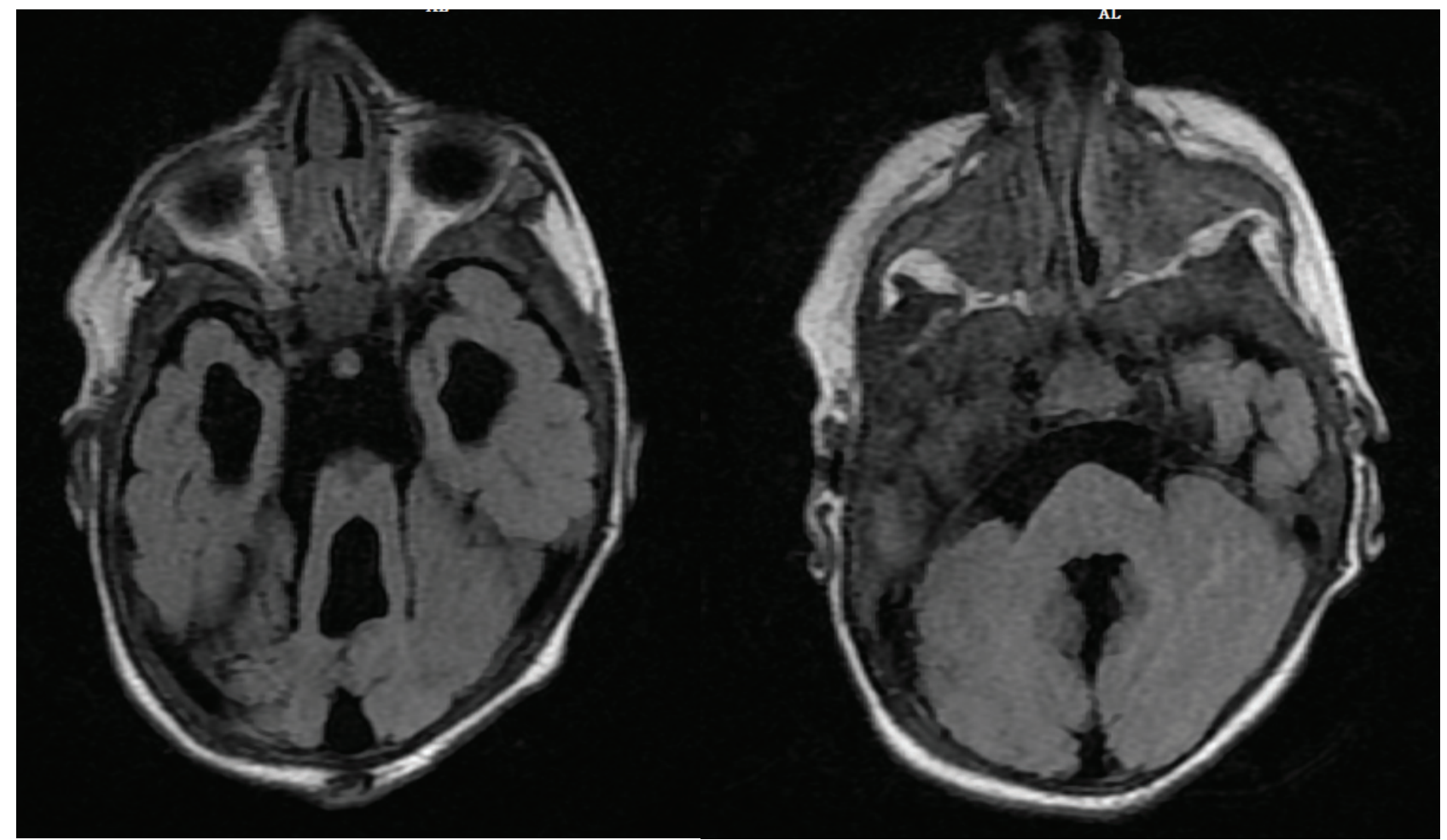

Figure 1: Axial T2 FLAIR image of the brain at the level of midbrain shows thickened and elongated superior cerebellar peduncles ("molar tooth" appearance,star mark)=(A)and, at the level of cerebellum shows vermian aplasia and an abnormally shaped fourth ventricle(star mark)(B). 


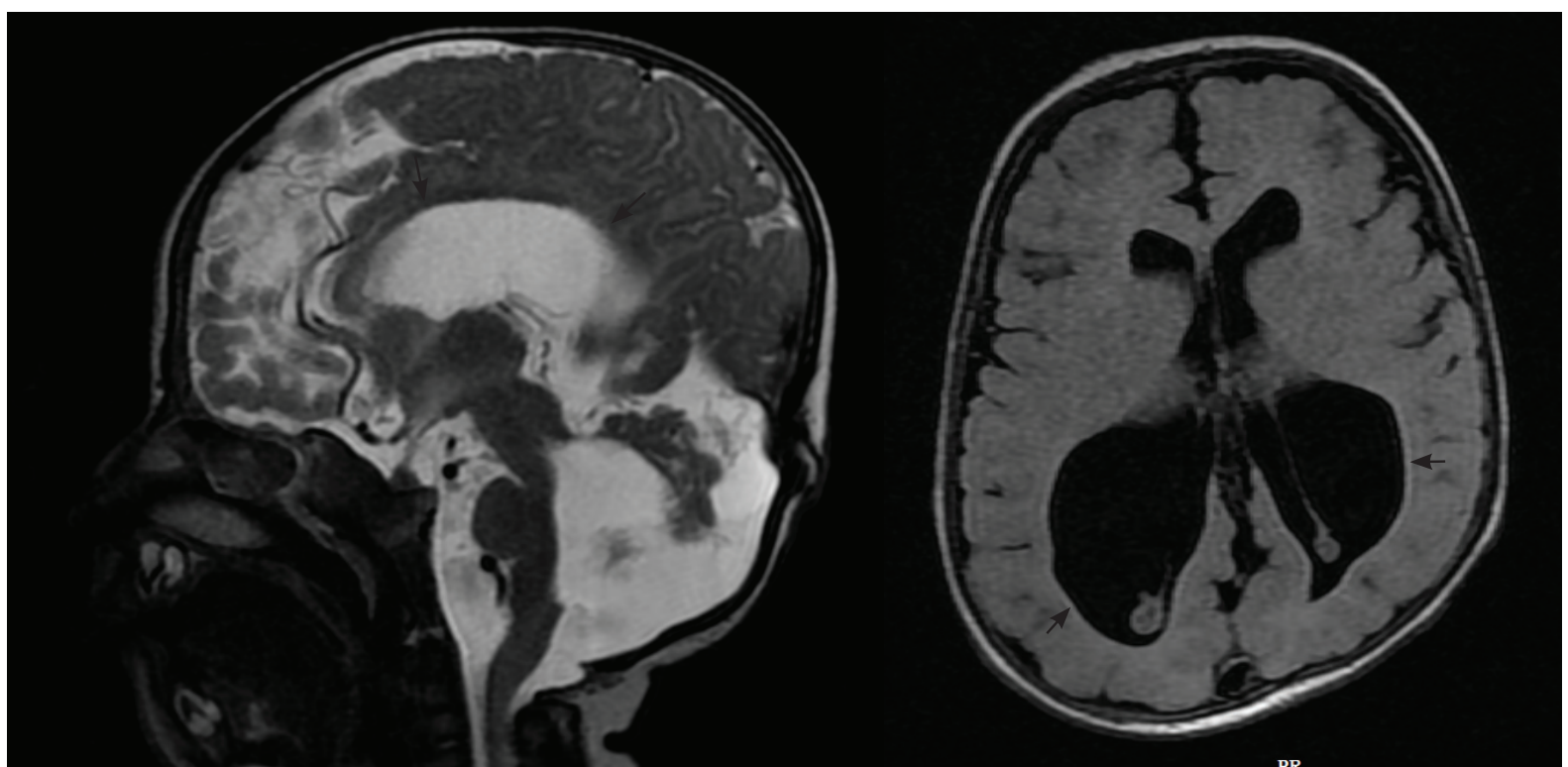

Figure 2(A and B): Sagittal T2 image shows corpus callosum dysgenesis with thinning and non-visualized splenium(black arrow)(A), Axial T2 FLAIR image shows dilated posterior half of lateral ventricles (colpocephaly) (black arrow), and, non-visualized Splenium of corpus callosum(B).

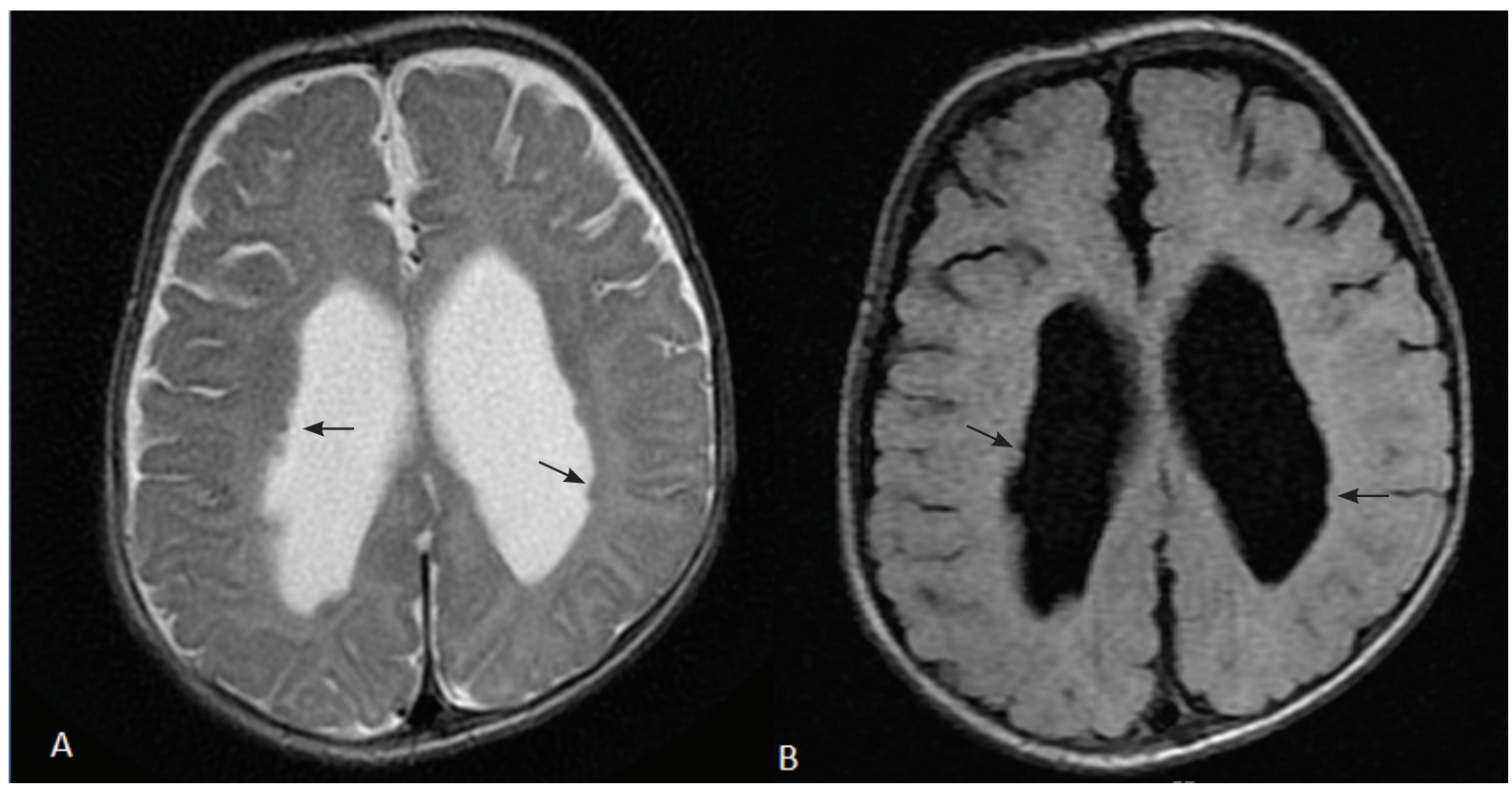

Figure 3 (A, B): Axial T2 and FLAIR images demonstrate periventricular nodular heterotopias(Black arrow) and Cerebral Atrophy

\section{Discussion}

JS is one of the rare malformations of the posterior fossa, largely defined by its classic "molar tooth" sign on axial cross-sectional imaging. On a molecular level, JS is thought to be a ciliopathy which results in abnormal signalling pathways involved in the development of cerebellum and other brain structures. ${ }^{3}$
Clinical manifestations are variable, with developmental delay, hypotonia, and breathing abnormalities being the most common presenting features. MRI is essential to clinch the diagnosis. ${ }^{2}$

While the "molar tooth" appearance (which results due to vermian dysplasia, abnormal superior cerebellar peduncles, and bizarre shape of the fourth ventricle) is essential for diagnosing JS, other finding should be 


\section{Mishra et al}

carefully looked for. Corpus callosum dysgenesis and colpocephaly are reported in $6-20 \%$ of cases. ${ }^{9}$ Relatively fewer studies have mentioned cortical atrophy and heterotopias as manifestations of JS.,10

$30 \%$ of the population of Joubert syndrome show renal abnormalties in the form of Cystic dysplasia with fetal lobulations and Juvenile type of nephronophthisis.

Management of JS/JSRD is primarily supportive, with prognosis depending heavily on the extent of involvement of other organs. Cerebral atrophy results in failure to thrive, delayed developmental milestones, and abnormal behaviour. When posterior cortex is predominantly involved, it may lead to progressive visuospatial dysfunction. Periventricular nodular heterotopias can serve as epileptogenic foci and result in focal drug resistant seizures. ${ }^{11}$ It is imperative that radiologists and clinicians focus on these additional findings as these may complicate an already complex line of management.

\section{Conflict of Interest: None \\ Source of Support: None}

\section{References}

1. Osborn AG, Hedlund GL, Salzman KL. Osborn's brain. Elsevier, Inc. 2017. p. 1191-1193.

2. Parisi MA. Clinical and molecular features of Joubert syndrome and related disorders. Am J Med Genet C Semin Med Genet. 2009;151C(4):326-340. https:// doi.org/10.1002/ajmg.c.30229

3. Parisi MA. The molecular genetics of Joubert syndrome and related ciliopathies: The challenges of genetic and phenotypic heterogeneity. Transl Sci Rare Dis. 2019;4(1-2):25-49. Published 2019 Jul 4. https:// doi.org/10.3233/TRD-190041.

4. Joubert M, Eisenring JJ, Robb JP, Andermann F. Familial agenesis of the cerebellar vermis. A syndrome of episodic hyperpnea, abnormal eye movements, ataxia, and retardation. Neurology. 1969;19(9):813825. https://doi.org/10.1212/wnl.19.9.813.

5. Bin Dahman HA, Bin Mubaireek AH, Alhaddad $\mathrm{ZH}$. Joubert syndrome in a neonate: case report with literature review. Sudan J Paediatr. 2016;16(1):53-57.

6. Zamponi N, Rossi B, Messori A, Polonara G, Regnicolo L, Cardinali C. Joubert syndrome with associated corpus callosum agenesis. Eur J Paediatr Neurol. 2002;6(1):63-66. https://doi.org/10.1053/ ejpn.2001.0542.

7. Bader I, Decker E, Mayr JA, et al. MKS1 mutations cause Joubert syndrome with agenesis of the corpus callosum. Eur J Med Genet. 2016;59(8):386-391. https://doi.org/10.1016/j.ejmg.2016.06.007.

8. Avery H. Weiss, Dan Doherty, Melissa Parisi, Dennis Shaw, Ian Glass, James O. Phillips; Eye Movement Abnormalities in Joubert Syndrome. Invest. Ophthalmol. Vis. Sci. 2009;50(10):4669-4677.https:// doi.org/10.1167/iovs.08-3299.

9. Harting, Inga \&Kotzaeridou, Urania \& Poretti, Andrea \& Seitz, A \&Pietz, J \&Bendszus, M \&Boltshauser, E. (2011). Interpeduncular Heterotopia in Joubert Syndrome: A Previously Undescribed MR Finding. AJNR. American journal of neuroradiology. 32. 1286-9. https://doi.org/10.3174/ajnr.A2488.

10. Poretti A, Huisman TA, Scheer I et-al. Joubert syndrome and related disorders: spectrum of neuroimaging findings in 75 patients. AJNR Am J Neuroradiol. 2011;32 (8): 1459-63. https:/doi. org/10.3174/ajnr.A2517.

11. Battaglia, G., Chiapparini, L., Franceschetti, S., Freri, E., Tassi, L., Bassanini, S., Villani, F., Spreafico, R., D'Incerti, L. and Granata, T. (2006), Periventricular Nodular Heterotopia: Classification, Epileptic History, and Genesis of Epileptic Discharges. Epilepsia, 47: 86-97. https://doi.org/10.1111/j.15281167.2006.00374.x 